J. Lake Sci. (湖泊科学) , 2016, 28(5): 1010-1017

DOI 10. 18307/2016. 0510

(c) 2016 by Journal of Lake Sciences

\title{
太湖流域滆湖底泥重金属赋存特征及其生物有效性
}

\author{
包先明 ${ }^{1}$, 晁建颖 ${ }^{2 * *}$, 尹洪斌 \\ (1: 淮北师范大学生命科学学院, 淮北 235000) \\ (2: 环境保护部南京环境科学研究所, 南京 210042) \\ (3: 中国科学院南京地理与湖泊研究所,南京 210008)
}

\begin{abstract}
摘 要: 为了探讨太湖流域滆湖底泥重金属 $(\mathrm{Cd} 、 \mathrm{Cr} 、 \mathrm{Cu} 、 \mathrm{Zn} 、 \mathrm{Ni}$ 和 $\mathrm{Pb})$ 的赋存特征及其生物有效性,对底泥重金属总量、 形态以及生物富集量进行了分析. 结果表明,6 种重金属含量的空间分布表现为北部湖区最高, 其次为南部湖区, 中部湖 区最低, 重金属 $\mathrm{Ni} 、 \mathrm{Cu} 、 \mathrm{Zn}$ 和 $\mathrm{Pb}$ 含量显著高于沉积物背景值, 分别是背景值的 4.77、3.89、2.96 和 2.76 倍, 重金属总量与 沉积物中的黏土成分含量具有显著相关性. 采用三级四部提取法对重金属形态进行分析表明,6 种重金属的生物有效态 (弱酸结合态、可还原态和可氧化态之和) 含量顺序为 $\mathrm{Cd}>\mathrm{Cu}>\mathrm{Zn}>\mathrm{Pb}>\mathrm{Ni}>\mathrm{Cr}$, 其中 $\mathrm{Cd} 、 \mathrm{Cu} 、 \mathrm{Zn}$ 和 $\mathrm{Pb}$ 的生物有效态含量分 别占总量的 $84.15 \% 、 78.47 \% 、 76.50 \%$ 和 $64.29 \% . \mathrm{Cu}$ 和 Zn 在铜锈环棱螺中富集含量要显著高于其他金属元素. 相关性分 析表明,6 种重金属中仅 $\mathrm{Cr}$ 和 $\mathrm{Pb}$ 的生物富集量与有效态含量具有显著相关性,这表明,重金属在生物体内的富集不仅与 有效态含量有关, 还与底泥重金属总量有关. 因此,评价滆湖重金属的生态风险时需要综合考虑重金属的总量及生物有 效态含量.
\end{abstract}

关键词: 沉积物; 重金属;赋存特征;生物有效性;滆湖;太湖流域;铜锈环棱螺

\section{Occurrence characteristics and bioavailability of heavy metals in surface sediments of Lake Gehu, Taihu Basin}

\author{
BAO Xianming ${ }^{1}$, CHAO Jianying ${ }^{2 * *} \&$ YIN Hongbin ${ }^{3}$ \\ (1: College of Life Science, Huaibei Normal University, Huaibei 235000, P.R.China) \\ (2: Nanjing Institute of Environmental Science, Ministry of Environmental Protection, Nanjing 210042, P.R. China) \\ (3: Nanjing Institute of Geography and Limnology, Chinese Academy of Sciences, Nanjing 210008, P.R.China)
}

\begin{abstract}
To investigate the occurrence characteristics and bioavailability of heavy metals $(\mathrm{Cd}, \mathrm{Cr}, \mathrm{Cu}, \mathrm{Zn}, \mathrm{Ni}$ and $\mathrm{Pb})$ in the sediments of Lake Gehu, total content, speciation and bioaccumulation of heavy metals were analyzed. Results showed that distribution of six heavy metals in the surface sediments indicated the highest degree of contamination in the north region, followed by the south region, and the lowest in the central region. $\mathrm{Ni}, \mathrm{Cu}, \mathrm{Zn}$ and $\mathrm{Pb}$ contents were significantly higher than background values, which were $4.77,3.89,2.96$ and 2.76 times higher than background values, respectively. Total heavy metal contents were significantly correlated with contents of clay particle. Speciation analysis of heavy metals showed that bioavailability fraction (sum of acid-extractable, reducible and oxidizable fractions) followed a descending order as: $\mathrm{Cd}>\mathrm{Cu}>\mathrm{Zn}>\mathrm{Pb}>\mathrm{Ni}>\mathrm{Cr}$. Bioavailability fraction contents of $\mathrm{Cd}, \mathrm{Cu}, \mathrm{Zn}$ and $\mathrm{Pb}$ were $84.15 \%, 78.47 \%, 76.50 \%$ and $78.47 \%$ of the total contents, respectively, which showed a relatively high potential ecological risk. $\mathrm{Cu}$ and $\mathrm{Zn}$ contents accumulated in Bellamya aeruginosa were significantly higher than other metal elements contents. Correlation analysis indicated that only $\mathrm{Cr}$ and $\mathrm{Pb}$ contents accumulated in Bellamya aeruginosa had significant correlation with bioavailability fraction contents; it turned out that the heavy metals contents accumulated in organism were not only related to the bioavailability fraction contents, but also related to total heavy metals contents in sediments. Therefore, it is necessary to consider total contents and bioavailability of heavy metals contents when we evaluate ecological risk of heavy metals in Lake Gehu.
\end{abstract}

* 国家水体污染控制与治理科技重大专项(2012ZX07103-005) 资助. 2015-08-16 收稿; 2015-10-28 收修改稿. 包 先明( 1978 ), 男, 博士, 副教授; E-mail:xmbao96@126.com.

** 通信作者; E-mail: njauchao@163.com. 
Keywords: Sediment; heavy metals; occurrence characteristics; bioavailability; Lake Gehu; Taihu Basin; Bellamya aeruginosa

重金属元素由于在环境中具有毒性大、易富集和难降解等特性而受到高度关注 ${ }^{[-2]}$. 重金属可通过地表 径流迁移、沉降,逐步转移至湖泊沉积物中,并可能在一定的环境条件下重新释放到上覆水体中,直接或间 接地对水生生物产生致毒致害作用,并通过生物富集、食物链放大等过程进一步影响人类健康 ${ }^{[3-4]}$. 因此,有 必要了解湖泊沉积物重金属的污染程度和特性. 研究表明, 不同形态的重金属具有不同的生物有效性和毒 性 ${ }^{[5]}$, 因此对沉积物重金属形态提取和分析对于辨识沉积物中重金属的污染状况和释放潜力具有重要的作 用. 底栖动物作为湖泊生态系统的重要组成部分, 长期生活于沉积物表层, 生活环境相对固定, 并且具有生 命周期长、迁移能力差、区域性强等特点, 对表层沉积物毒性及污染状况具有直接的指示作用 ${ }^{[6-7]}$. 因此, 研 究重金属在湖泊不同介质(沉积物和底栖生物) 中的赋存特征对于全面掌握了解重金属迁移规律以及对生 物的毒害作用等十分必要.

滆湖 $\left(31^{\circ} 29^{\prime} \sim 31^{\circ} 42^{\prime} \mathrm{N}, 119^{\circ} 44^{\prime} \sim 119^{\circ} 53^{\prime} \mathrm{E}\right.$ ) 俗称西太湖, 是苏南地区的第二大湖. 位于长江三角洲太湖 流域西部, 常州市武进区西南部. 湖泊总面积为 $164 \mathrm{~km}^{2}$. 滆湖是典型的浅水湖泊,具有饮用水后备水源、旅 游休闲、水产养殖和蓄洪灌溉等多种使用功能 ${ }^{[8]}$. 近几年来随着滆湖周围地区经济的迅速发展和城市化进 程的加快, 滆湖流域污染负荷不断增加, 滆湖水体污染程度也日益加剧, 已经严重影响到水生生态环境, 长 期生活在滆湖底泥表层的底栖生物也难免受到湖泊污染的影响. 在滆湖底栖生物中, 铜锈环棱螺 (Bellamya aeruginosa) 的分布较为广泛 ${ }^{[9]}$, 其作为以摄食沉积物中有机碎屑、细菌和藻类为生的底栖软体动物, 具备分 布广、个体大小适中、移动性差、适应性强等特点 ${ }^{[10]}$, 同时也被人类食用, 在食物链中具有重要作用, 能够反 映所在水生生态系统的重金属污染特征.

目前, 针对滆湖沉积物的研究主要集中在营养盐以及水生生物方面 ${ }^{[1-12]}$, 而对沉积物重金属的研究较 ${ }^{\text {少 }}{ }^{[13]}$, 尤其缺乏重金属的生物有效性以及在底栖生物中的富集特征的研究. 本文以滆湖为研究对象, 测定 表层沉积物中 $\mathrm{Cd} 、 \mathrm{Cr} 、 \mathrm{Cu} 、 \mathrm{Zn} 、 \mathrm{~Pb}$ 和 $\mathrm{Ni} 6$ 种重金属元素的总量、形态以及在铜锈环棱螺中的富集含量, 并通 过相关性分析, 对滆湖表层沉积物重金属的生物有效性进行探讨, 以期为探明滆湖沉积物中重金属污染状 况及污染特征提供基础数据.

\section{1 材料与方法}

\section{1 样品采集}

于 2014 年 10 月在滆湖采集底泥和底栖生物样品, 全湖共 布设 9 个采样点(图 1), 分别为北部湖区 $(G 1 、 G 2 、 G 3)$ 、中部 湖区 $(G 4 、 G 5 、 G 6)$ 和南部湖区 $(G 7 、 G 8 、 G 9)$. 其中, 湖区划分 主要依据湖泊水质状况, 北部湖区和中部湖区因人湖河流污 染物输人以及湖区水产养殖等问题导致水质相对较差 ${ }^{[8]}$, 而 南部狭长湖区水生植被覆盖率较高, 水质相对较好. 采用彼得 森采泥器采集表层 $10 \mathrm{~cm}$ 的沉积物样品, 将采集的表层沉积物 样品混合均匀后装人聚乙烯自封袋中密封, 低温保存送回实 验室进行预处理及分析. 同样采用彼得森采泥器采集底栖动 物 (铜锈环棱螺), 每个样点采集 6 次混合成一个样品, 然后经 过 60 目尼龙篮网现场篮洗,剩余物带回实验室进行分拣.

\section{2 样品处理及分析方法}

采集的沉积物样品经冷冻干燥机冷冻干燥后,去掉杂物 及石块, 经玛瑙研钵研磨处理, 过 200 目尼龙篮,它存备用. 采 集的底栖样品置人白色盘中, 将铜锈环棱螺活体挑出, 置于冷 Fig.1 Location of sampling sites in Lake Gehu 冻干燥机内冷冻干燥, 之后在无菌操作台上进行解剖, 用剪刀 将铜锈环棱螺组织剪碎, 然后采用玛瑙研针研磨至粉末状, 贮存备用. 沉积物重金属形态提取是采用欧共体 
标准物质局提出的三级四步提取法 ${ }^{[14]}$, 简称 BCR 法, 将重金属形态分为弱酸提取态、可还原态、可氧化态和 残渣态, 前 3 种形态由于其生物可利用性统称为生物有效态 ${ }^{[15-16]}$. 沉积物样品、生物样品和各形态提取液中 重金属元素含量利用电感耦合等离子体质谱仪 ( ICP-MS, Agilent 7700cx 型) 测定 ${ }^{[17]}$. 为保证分析的准确性, 同步分析了由国家地质实验测试中心生产的湖底沉积物顺序提取微量元素标准物质 (GBW07436), 各重金 属元素不同形态回收率在 $94.1 \% \sim 119.9 \%$ 范围内,符合美国 EPA 标准要求的 $80 \% \sim 120 \%$ 的范围.

沉积物总有机碳 (TOC) 含量采用重铬酸钾容量法测定 ${ }^{[18]}$; 沉积物粒度使用英国 Malvern 公司的 Mastersizer 2000 型激光粒度分析仪进行测定 ${ }^{[19]}$, 其中粒度 $<2.00 \mu \mathrm{m}$ 为黏土, 粒度在 $2.00 \sim 63.00 \mu \mathrm{m}$ 之间为粉砂, 粒度 $>63.00 \mu \mathrm{m}$ 为砂砾.

\section{2 结果与讨论}

\section{1 表层沉积物理化性质及重金属分布}

沉积物是重金属等环境污染物的源和汇, 沉积物粒度和总有机碳含量在一定程度上能够影响对重金属 的吸附、解吸以及重金属在环境中的迁移 ${ }^{[20]}$. 滆湖表层沉积物中黏土含量范围为 $10.11 \% \sim 14.82 \%$, 粉砂含 量范围为 $80.98 \% \sim 88.11 \%$, 砂砾含量范围为 $1.57 \% \sim 6.62 \%$, 从黏土含量的空间分布来看, 北部湖区 >中部湖 区>南部湖区, 平均含量分别为 $13.57 \% 、 11.31 \%$ 和 $11.09 \%$. TOC 含量范围为 $4.31 \% \sim 15.43 \%$, 南部湖区最高, 平均含量为 $13.59 \%$, 其次为北部湖区, 平均含量为 $7.68 \%$, 中部湖区最低, 平均含量为 $5.28 \% \mathrm{mg} / \mathrm{kg}$ (表 1 ). 6 种重金属 $(\mathrm{Cu} 、 \mathrm{Zn} 、 \mathrm{~Pb} 、 \mathrm{Cd} 、 \mathrm{Ni}$ 和 $\mathrm{Cr})$ 的含量范围分别为 $31.02 \sim 82.03 、 90.24 \sim 185.73 、 25.50 \sim 63.36 、 0.25 \sim 0.75$ 、 $38.29 \sim 84.05$ 和 71.99 168.32 mg/ kg (表 1), 6 种重金属均表现为北部湖区重金属含量最高, 分别是沉积物 背景值的 $3.89 、 2.76 、 2.96 、 2.44 、 4.77$ 和 1.91 倍, 中部湖区和南部湖区差异较小, 南部湖区的 $\mathrm{Cu} 、 \mathrm{Zn} 、 \mathrm{~Pb} 、 \mathrm{Cd} 4$ 种金属含量略高于中部湖区, 中部湖区的 $\mathrm{Ni}$ 和 $\mathrm{Cr}$ 略高于南部湖区. 从金属污染程度来看, 3 个湖区均呈现 出 $\mathrm{Ni}$ 的超标倍数最高, 其次是 $\mathrm{Cu}$. 滆湖北部上游及周围地区城镇化进程的加快和工业企业的快速发展, 生 活污水和工业废水大量进入湖内是导致滆湖北部重金属污染的重要原因.

表 1 滆湖沉积物理化性质及重金属含量

Tab. 1 Sediment properties and heavy metal contents in sediments of Lake Gehu

\begin{tabular}{lccccccccccc}
\hline 区域 & 点位 & TOC/\% & 黏土/\% & 粉砂/\% & 砂砾 $/ \%$ & $\begin{array}{c}\mathrm{Cu} / \\
(\mathrm{mg} / \mathrm{kg})\end{array}$ & $\begin{array}{c}\mathrm{Zn} / \\
(\mathrm{mg} / \mathrm{kg})\end{array}$ & $\begin{array}{c}\mathrm{Pb} / \\
(\mathrm{mg} / \mathrm{kg})\end{array}$ & $\begin{array}{c}\mathrm{Cd} / \\
(\mathrm{mg} / \mathrm{kg})\end{array}$ & $\begin{array}{c}\mathrm{Ni} / \\
(\mathrm{mg} / \mathrm{kg})\end{array}$ & $\begin{array}{c}\mathrm{Cr} / \\
(\mathrm{mg} / \mathrm{kg})\end{array}$ \\
\hline 北部湖区 & $\mathrm{G} 1$ & 7.63 & 12.35 & 81.03 & 6.62 & 82.03 & 154.21 & 55.87 & 0.75 & 84.05 & 168.32 \\
& $\mathrm{G} 2$ & 7.45 & 14.82 & 80.98 & 4.20 & 74.66 & 185.73 & 63.36 & 0.68 & 75.93 & 136.00 \\
& $\mathrm{G} 3$ & 7.96 & 13.54 & 82.47 & 3.99 & 64.14 & 150.38 & 54.16 & 0.55 & 64.65 & 150.01 \\
中部湖区 & $\mathrm{G} 4$ & 5.47 & 12.93 & 84.97 & 2.10 & 43.89 & 120.95 & 30.88 & 0.41 & 58.93 & 98.60 \\
& $\mathrm{G} 5$ & 4.31 & 10.23 & 83.72 & 6.05 & 31.02 & 90.24 & 25.50 & 0.25 & 46.20 & 82.30 \\
& $\mathrm{G} 6$ & 6.06 & 10.11 & 86.91 & 2.99 & 36.94 & 100.58 & 35.33 & 0.43 & 38.29 & 77.80 \\
南部湖区 & $G 7$ & 10.36 & 11.03 & 87.64 & 2.10 & 50.78 & 125.21 & 40.54 & 0.39 & 50.98 & 92.56 \\
& $G 8$ & 14.98 & 10.32 & 88.11 & 1.57 & 40.72 & 111.05 & 37.42 & 0.53 & 40.65 & 81.27 \\
& $G 9$ & 15.43 & 12.58 & 85.35 & 2.07 & 35.45 & 102.76 & 36.57 & 0.42 & 43.47 & 71.99 \\
背景值 & & - & - & - & - & 18.90 & 59.20 & 19.50 & 0.27 & 15.70 & 79.30 \\
\hline
\end{tabular}

\section{2 沉积物中重金属与有机质和各颗粒含量的相关性}

滆湖沉积物中的黏土含量与 $\mathrm{Zn}$ 含量呈极显著相关 $(P<0.01)$, 与 $\mathrm{Cu} 、 \mathrm{~Pb} 、 \mathrm{Cd} 、 \mathrm{Ni}$ 和 $\mathrm{Cr}$ 呈显著相关 $(P<$ $0.05)$, 这表明, 重金属易于被沉积物中的黏土成分吸附, 该分析结果与以往研究发现的沉积物中重金属与 黏土成分具有相关性 ${ }^{[21]}$ 一致. 相关性分析 (表 2) 发现, 沉积物中重金属含量与总有机碳含量之间的相关性 较差,其原因可能是由于人类活动比较频繁,导致人湖污染物较为复杂,影响了相关性.

\section{3 表层沉积物中重金属各形态的分布}

滆湖不同湖区表层沉积物中重金属各形态的分布如图 2 所示. 重金属 $\mathrm{Cr}$ 和 $\mathrm{Ni}$ 主要以残渣态存在,平均 
表 2 滆湖沉积物理化性质与重金属含量之间的相关性

Tab.2 Pearson's correlation between sediment properties and metal concentrations in Lake Gehu

\begin{tabular}{|c|c|c|c|c|c|c|c|c|c|c|}
\hline & TOC & 黏土 & 粉砂 & 砂砾 & $\mathrm{Cu}$ & $\mathrm{Zn}$ & $\mathrm{Pb}$ & $\mathrm{Cd}$ & $\mathrm{Ni}$ & $\mathrm{Cr}$ \\
\hline TOC & 1.000 & -0.066 & 0.447 & -0.581 & -0.161 & -0.137 & 0.018 & 0.106 & -0.329 & -0.302 \\
\hline 黏土 & & 1.000 & $-0.711^{*}$ & 0.112 & $0.663^{*}$ & $0.813^{* *}$ & $0.718^{*}$ & 0.570 * & $0.726^{*}$ & $0.630^{*}$ \\
\hline 粉砂 & & & 1.000 & $-0.773^{*}$ & $-0.730^{*}$ & $-0.700 *$ & -0.662 & -0.572 & $-0.856^{* *}$ & $-0.812^{* *}$ \\
\hline 砂砾 & & & & 1.000 & 0.477 & 0.294 & 0.320 & 0.294 & 0.591 & 0.607 \\
\hline $\mathrm{Cu}$ & & & & & 1.000 & $0.932^{* *}$ & $0.929^{\text {** }}$ & $0.893^{\text {*** }}$ & $0.941^{\text {*** }}$ & $0.947^{\text {** }}$ \\
\hline $\mathrm{Zn}$ & & & & & & 1.000 & $0.946^{\text {** }}$ & $0.829^{* *}$ & $0.874^{* * *}$ & $0.841^{\text {** }}$ \\
\hline $\mathrm{Pb}$ & & & & & & & 1.000 & $0.890^{\text {*** }}$ & $0.799^{* * *}$ & 0.837 ** \\
\hline Cd & & & & & & & & 1.000 & $0.776^{*}$ & $0.803^{* *}$ \\
\hline $\mathrm{Ni}$ & & & & & & & & & 1.000 & $0.938^{* *}$ \\
\hline $\mathrm{Cr}$ & & & & & & & & & & 1.000 \\
\hline
\end{tabular}

** 表示相关系数在 0.01 水平上显著, $*$ 表示相关系数在 0.05 水平上显著.
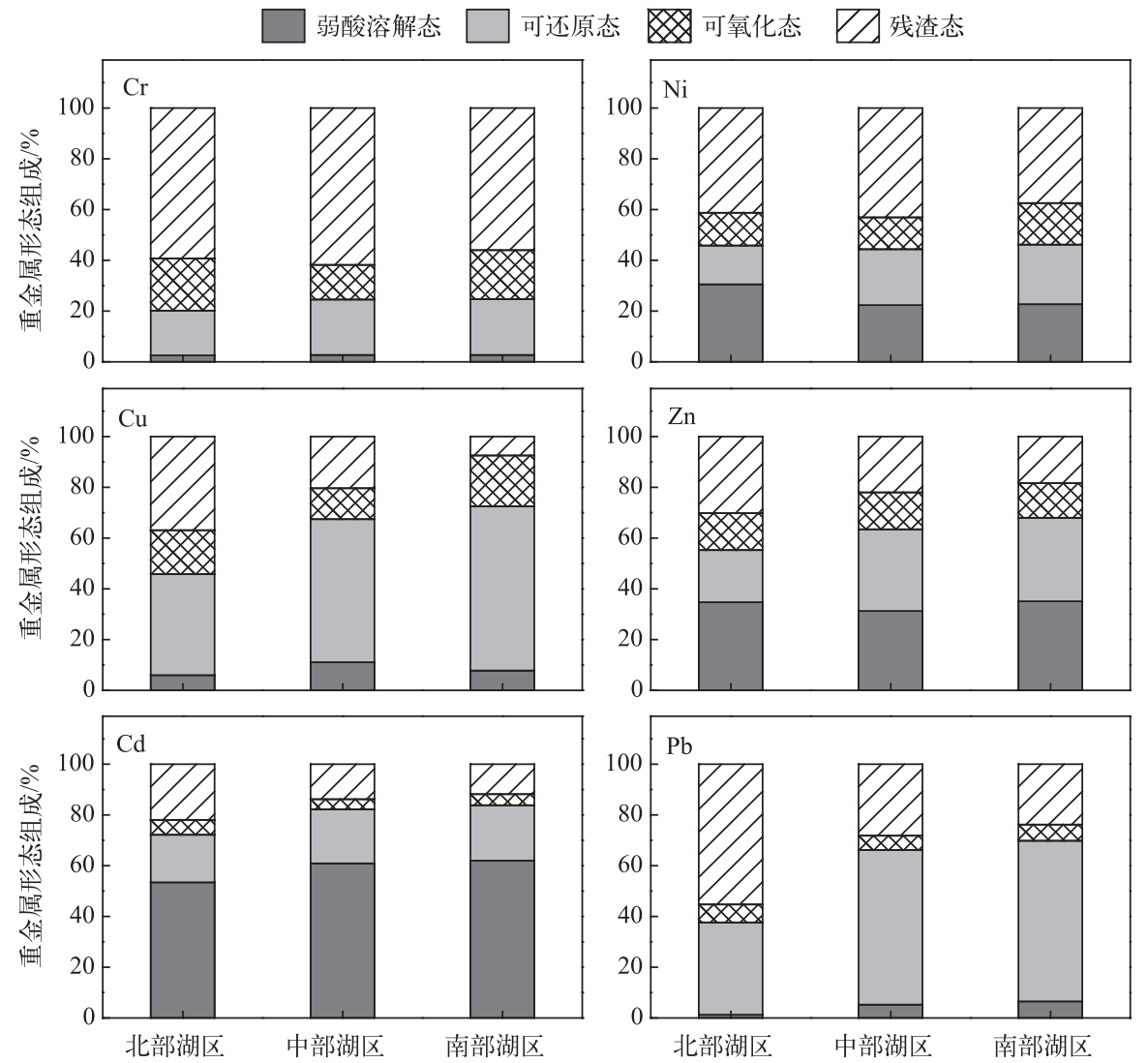

图 2 滆湖表层沉积物中重金属各形态的分布特征

Fig.2 Distribution characteristics of heavy metals forms in surface sediments of Lake Gehu

含量占总量的比值分别为 $59.04 \%$ 和 $40.53 \%$. 有研究认为残渣态的重金属离子易结合在土壤硅铝酸盐矿物 晶格中, 性质较为稳定,一般情况下难以释放, 对沉积物中重金属的迁移和生物可利用性贡献小 ${ }^{[22]}$, 因此, $\mathrm{Cr}$ 和 $\mathrm{Ni}$ 对环境和生物是比较安全的. $\mathrm{Cd}$ 元素以弱酸结合态为主, 平均含量为 $58.84 \%$, 其次为可还原态, 平均 
含量为 $20.61 \%$, 研究表明, Cd 元素在沉积物中易吸附在细颗粒表面,在碳酸盐矿物形成的过程中, Cd 易与 $\mathrm{Ca}^{2+}$ 发生替代反应 ${ }^{[23]}$, 因而对 $\mathrm{pH}$ 值的敏感度较高, 在酸性条件下容易释放, 进而对环境和生物可能产生危 害和毒性, 这表明, $\mathrm{Cd}$ 和 $\mathrm{Zn}$ 均具有较高的潜在释放风险. 与 $\mathrm{Cd}$ 类似, $\mathrm{Zn}$ 元素的主要形态为弱酸结合态, 平 均含量为 $33.70 \%$, 其次为可还原态, 平均含量为 $28.60 \%$. Cu 和 Pb 以可还原态为主, 含量分别达到 $53.63 \%$ 和 $53.60 \%$, 可还原态属于较强的离子键结合的化学形态, 通常不易释放, 但当水体中氧化还原电位降低或 水体严重缺氧情况下,这种结合形态的重金属键被还原, 可能对水体造成二次污染.

重金属生物有效态比例越高, 表明沉积物中重金属越易释放并造成二次污染, 其生物有效性也就越 大 ${ }^{[24]} .6$ 种重金属的生物有效态含量顺序为 $\mathrm{Cd}>\mathrm{Cu}>\mathrm{Zn}>\mathrm{Pb}>\mathrm{Ni}>\mathrm{Cr}$, 其中 $\mathrm{Cd} 、 \mathrm{Cu} 、 \mathrm{Zn}$ 和 $\mathrm{Pb}$ 的生物有效态含量 均大于 $60 \%$, 分别占总量的 $84.15 \% 、 78.47 \% 、 76.50 \%$ 和 $64.29 \%$. 这表明, 沉积物中 $\mathrm{Cd} 、 \mathrm{Cu} 、 \mathrm{Zn}$ 和 $\mathrm{Pb}$ 有较高的 生物有效性,因而在环境中具有较高的释放风险.

\section{4 重金属在铜锈环棱螺体内的富集}

6 种重金属在滆湖不同湖区铜锈环棱螺体内的含量呈现不同分布特征, 北部湖区为 $\mathrm{Zn}>\mathrm{Cu}>\mathrm{Cr}>\mathrm{Ni}>\mathrm{Pb}>$ $\mathrm{Cd}$, 中部湖区为 $\mathrm{Zn}>\mathrm{Cu}>\mathrm{Ni}>\mathrm{Cr}>\mathrm{Pb}>\mathrm{Cd}$, 南部湖区为 $\mathrm{Zn}>\mathrm{Cu}>\mathrm{Pb}>\mathrm{Ni}>\mathrm{Cr}>\mathrm{Cd}$. 总体而言, 滆湖 3 个湖区均呈现 出 $\mathrm{Zn}$ 在铜锈环棱螺体内富集的含量最高, 其次是 $\mathrm{Cu}$ (图 3).

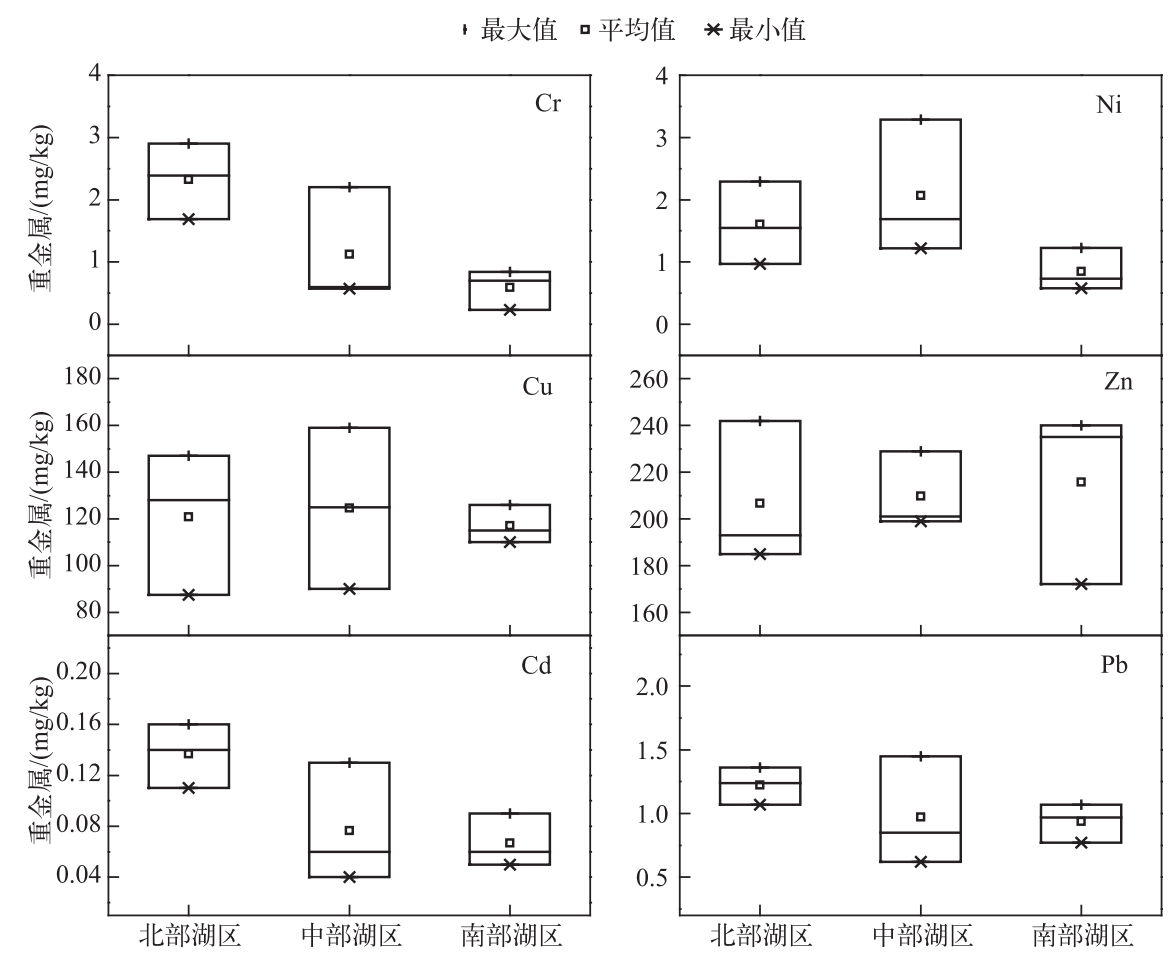

图 3 滆湖不同湖区重金属在铜锈环棱螺体内的富集含量

Fig. 3 Contents of heavy metals in Bellamya aeruginosa from different zones in Lake Gehu

为了比较水生生物对沉积物中不同污染物的积累能力, 一般用生物-沉积物积累因子 (biota-sediment accumulation factor, BSAF) 来表示 ${ }^{[25]}$. 表 3 结果显示, 铜锈环棱螺对沉积物中 6 种重金属的积累水平存在较大 差异,其中 $\mathrm{Cu}$ 和 $\mathrm{Zn}$ 的积累量明显高于其他元素,这与 Liang 等 ${ }^{[26]}$ 和李丽娜等 ${ }^{[27]}$ 的研究结果基本一致.

滆湖中 $\mathrm{Cr}$ 的生物积累量及沉积物中 $\mathrm{Cr}$ 的总量及有效态含量均呈极显著正相关 $(P<0.01) ; \mathrm{Pb}$ 的生物积 累量与 $\mathrm{Pb}$ 的有效态含量呈极显著正相关 $(P<0.01)$, 而且也与 $\mathrm{Pb}$ 总量显著相关 $(P<0.05) ; \mathrm{Cd}$ 和 $\mathrm{Ni}$ 的生物 积累量与其在沉积物中的总量及有效态含量相关性不显著; $\mathrm{Cu}$ 和 $\mathrm{Zn}$ 的生物积累与其形态和总量均没有显 著相关性,这表明 $\mathrm{Cr}$ 和 $\mathrm{Pb}$ 的有效态含量可以较好地指示重金属的生物可利用性(图 4). 
表 3 铜锈环棱螺对滆湖污染沉积物中重金属的生物-沉积物积累因子"

Tab.3 Biota-sediment accumulation factors of Bellamya aeruginosa to heavy metals in the sediments of Lake Gehu

\begin{tabular}{lccccccc}
\hline 区域 & 点位 & $\mathrm{Cu}$ & $\mathrm{Zn}$ & $\mathrm{Pb}$ & $\mathrm{Cd}$ & $\mathrm{Ni}$ & $\mathrm{Cr}$ \\
\hline 北部湖区 & $\mathrm{G} 1$ & 1.56 & 1.57 & 0.02 & 0.21 & 0.02 & 0.01 \\
& $\mathrm{G} 2$ & 1.97 & 1.04 & 0.02 & 0.21 & 0.03 & 0.02 \\
& $\mathrm{G} 3$ & 1.36 & 1.23 & 0.02 & 0.20 & 0.02 & 0.01 \\
中部湖区 & $\mathrm{G} 4$ & 3.62 & 1.89 & 0.03 & 0.32 & 0.06 & 0.01 \\
& $\mathrm{G} 5$ & 2.90 & 2.21 & 0.06 & 0.24 & 0.04 & 0.03 \\
南部湖区 & $\mathrm{G} 6$ & 3.38 & 2.00 & 0.02 & 0.09 & 0.03 & 0.01 \\
& $\mathrm{G} 7$ & 2.26 & 1.88 & 0.03 & 0.23 & 0.01 & 0.01 \\
& $\mathrm{G} 8$ & 2.70 & 1.55 & 0.02 & 0.09 & 0.02 & 0.00 \\
& $\mathrm{G} 9$ & 3.55 & 2.34 & 0.03 & 0.14 & 0.03 & 0.01 \\
\hline
\end{tabular}

* 生物-沉积物积累因子为处理组生物体中重金属含量与沉积物中重金属含量之比.
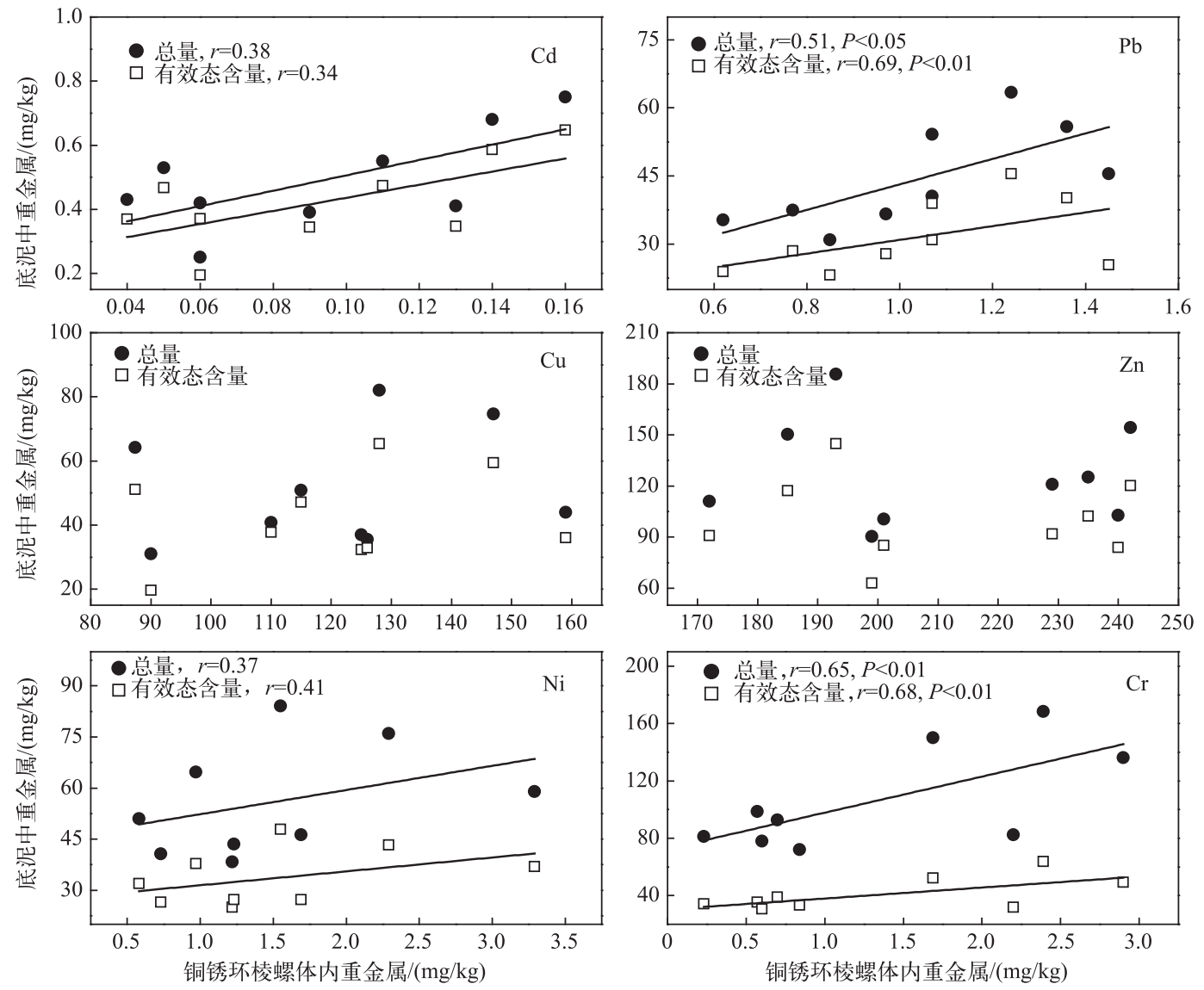

图 4 滆湖重金属在铜锈环棱螺体内的富集量与沉积物中重金属总量、有效态含量的相关性

Fig.4 Correlation between total and metal concentrations in sediment and metal concentrations in tissue of Bellamya aeruginosa in Lake Gehu

尽管 $\mathrm{Cu}$ 的有效态含量与在铜锈环棱螺体内的富集含量没有显著相关性, 但其在生物体内具有较高的 
生物积累量,应给予足够的重视. 同时,研究发现 6 种重金属中 Cd 的生物有效态含量最高,但在铜锈环棱螺 中的富集量较低,这是由于生物对重金属的富集不仅与重金属的生物有效性有关,还与环境中金属元素的 总量以及生物的生理特征有关.

\section{3 参考文献}

[ 1 ] Akcay H, Oguz A, Karapire C. Study of heavy metal pollution and speciation in Buyak Menderes and Gediz river sediments. Water Research, 2003, 37: 813-822.

[ 2 ] Fujita M, Ide Y, Sato D et al. Heavy metal contamination of coastal lagoon sediments: Fongafale Islet, Funafuti Atoll, Tuvalu. Chemosphere, 2014, 95: 628-634.

[ 3 ] Zhu HN, Yuan XZ, Zeng GM et al. Ecological risk assessment of heavy metals in sediments of xiawan port based on modified potential ecological risk index. Transactions of Nonferrous Metals Society of China, 2012, 22(6) : 1470-1477.

[ 4 ] Yu RL, Yuan X, Zhao YH et al. Heavy metal pollution in intertidal sediments from Quanzhou Bay, China. Journal of Environmental Sciences, 2008, 20(6) : 664-669.

[ 5 ] Yang ZF, Wang Y, Shen ZY et al. Distribution and speciation of heavy metals in sediments from the mainstream, tributaries, and lakes of the Yangtze River Catchment of Wuhan, China. Journal of Hazardous Materials, 2009, 166 (2): 1186-1194.

[6] Lu Qiang, Chen Huili, Shao Xiaoyang et al. Ecological characteristics of macrobenthic communities and its relationships with environmental factors in Hangzhou Xixi Wetland. Acta Ecologica Sinica, 2013, 33(9) : 2803-2815 (in Chinese with English abstract). [ 陆强, 陈慧丽, 郡晓阳等. 杭州西溪湿地大型底栖动物群落特征及与环境因子的关系. 生态学 报, 2013, 33(9): 2804-2815.]

[ 7 ] Pedersen ML, Friberg N, Skriver J et al. Restoration of Skjern Riverand its valley-short-term effects on river habitats, macrophytes and macroinvertebrates. Ecological Engineering, 2007, 30(2) : 145-156.

[ 8 ] Tao Hua, Pan Jizheng, Shen Yaoliang et al. Overview and degradation reasons of submerged macrophytes of Gehu Lake. Environmental Science and Technology, 2010, 23(5) : 64-68(in Chinese with English abstract).[ 陶花, 潘继征, 沈耀良 等. 滆湖沉水植物概况及退化原因分析. 环境科技, 2010, 23(5): 64-68.]

[ 9 ] Wang Liqing, Wu Liang, Zhang Ruilei et al. Spatiotemporal variation of zoobenthos community and bio-assessment of water quality in Gehu Lake. Chinese Journal of Ecology, 2012, 31( 8) : 1990-1996( in Chinese with English abstract). [ 王丽 卿, 吴亮, 张瑞雷等. 滆湖底栖动物群落的时空变化及水质生物学评价. 生态学杂志, 2012, 31(8): 1990-1996.]

[10] Ma Taowu, Zhu Cheng, Wang Guiyan et al. Bioaccumulation of sediment heavy metals in Bellamya aeruginosa and its relations with the metals geochemical fractions. Chinese Journal of Applied Ecology, 2010, 21(3) : 734-742(in Chinese with English abstract). [马陶武, 朱程, 王桂岩等. 铜锈环棱螺对沉积物中重金属的生物积累及其与重金属赋存形态的 关系. 应用生态学报, 2010, 21(3): 734-742.]

[11] Tao Hua, Pan Jizheng, Shen Yaoliang et al. Effects of substrate character of Lake Gehu on the growth of Potamogeton crispus and Ebdea natalii. J Lake Sci, 2011, 23(3) : 383-388 (in Chinese with English abstract). DOI: 10.18307/2011. 0310. [ 陶花, 潘继征, 沈耀良等. 滆湖底质特性对菹草 (Potamogeton crispus) 和伊乐藻 (Elodea nattalii) 生长的影响. 湖泊科学, 2011, 23(3): 383-388.]

[12] Chen Lijing, Peng Ziran, Kong Youjia et al. Characteristics of community structure of planktonic algae in Gehu Lake of Jiangsu Province. Chinese Journal of Ecology, 2008, 27(9) : 1549-1556(in Chinese with English abstract). [ 陈立婧, 彭 自然, 孔优佳等. 江苏滆湖浮游藻类群落结构特征. 生态学杂志, 2008, 27(9): 1549-1556.]

[13] Yin HB, Cai YJ, Duan HT et al. Use of DGT and conventional methods to predict sediment metal bioavailability to a field inhabitant freshwater snail (Bellamya aeruginosa) from Chinese eutrophic lakes. Journal of Hazardous Materials, 2014, 264: 184-194.

[14] Ure AM, Quevauviller PH, Muntau H et al. Speciation of heavy metals in soils and sediments. An account of the improvement and harmonization of extraction techniques undertaken under the auspices of the BCR of the commission of the European Communities. International Journal of Environmental Analytical Chemistry, 1993, 51(1/2/3/4) : 135-151.

[15] Zhang Fengying, Yan Baixing, Zhu Lilu. Speciation of heavy metals in sediment of the Songhua River, northeast of China. Journal of Agro-Environment Science, 2010, 29(1): 163-167( in Chinese with English abstract). [张凤英, 阎百兴, 朱立 
禄. 松花江沉积物重金属形态赋存特征研究. 农业环境科学学报, 2010, 29(1):162-167.]

[16] Jiao Wei, Lu Shaoyong, Niu Yong et al. Distribution of bio-available heavy metals in river sediments of Lake Taihu. Environmental Chemistry, 2013, 32(12) : 2315-2320(in Chinese with English abstract).[焦伟, 卢少勇, 牛勇等. 环太湖河 流沉积物中生物有效态重金属分布. 环境化学, 2013, 32(12) : 2315-2320.]

[17] Kong Ming, Dong Zenglin, Chao Jianying et al. Bioavailability and ecological risk assessment of heavy metals in surface sediments of Lake Chaohu. China Environmental Science, 2015, 35(4) : 1223-1229(in Chinese with English abstract). [孔明, 董增林, 晁建颖等. 巢湖表层沉积物重金属生物有效性与生态风险评价. 中国环境科学, 2015, 35(4): 1223-1229.]

[18] Institute of Soil Science, Chinese Academy of Sciences ed. Soil physical and chemical analyses. Shanghai: Shanghai Scientific and Technical Publishers, 1978: 62-67(in Chinese).[中国科学院南京土壤研究所. 土壤理化分析. 上海: 上海 科学技术出版社, 1978: 62-67.]

[19] Qiang Mingrui, Chen Fahu, Zhou Aifeng et al. Preliminary study on dust storm events documented by grain size component of Sugan Lake sediments, north Qaidam basin. Quaternary Science, 2006, 26(6) : 915-922(in Chinese with English abstract). [强明瑞, 陈发虎, 周爱锋等. 苏干湖沉积物粒度组成记录尘暴事件的初步研究. 第四纪研究, 2006,26 (6) : 915-922.]

[20] Zhang LP, Ye X, Feng H et al. Heavy metal contamination in western Xiamen Bay sediments and its vicinity, China. Marine Pollution Bulletin, 2007, 54(7): 974-982.

[21] Zhang Lei, Qin Yanwen, Zheng Binghui et al. Distribution and pollution assessment of heavy metals in sediments from typical areas in the Bohai sea. Acta Scientiae Circumstantiae, 2011, 31(8): 1676-1684(in Chinese with English abstract). [张雷, 秦延文, 郑丙辉等. 环渤海典型海域潮间带沉积物中重金属分布特征及污染评价. 环境科学学报, 2011, 31(8): 1676-1684.]

[22] Teasdale PR, Apte SC, Ford PW et al. Geochemical cycling and speciation of copper in waters and sediments of macquarie harbour, Western Tasmania. Estuarine, Coastal and Shelf Science, 2003, 57(3) : 475-487.

[23] Liu Enfeng, Shen Ji, Zhu Yuxin. Determination of heavy metal chemical forms by BCR method for Taihu Lake sediments. Research of Environmental Scinece, 2005, 18(2) : 57-60(in Chinese with English abstract).[刘恩峰, 沈吉, 朱育新. 重 金属元素 BCR 提取法及在太湖沉积物研究中的应用. 环境科学研究, 2005, 18(2): 57-60.]

[ 24] He Jiang, Wang Xinwei, Li Chaosheng et al. Pollution character of heavy metals in eater-sediment system from Baotou section of the Yelloe river. Acta Scientiae Circumstantiae, 2003, 23(1): 53-57( in Chinese with English abstract). [ 何江, 王 新伟, 李朝胜等. 黄河包头段水-沉积物系统中重金属的污染特征. 环境科学学报, 2003, 23(1): 53-57.]

[25] Gillis PL, Dixon DG, Borgmann U et al. Uptake and depuration of cadmium, nickel, and lead in laboratory exposed Tubifex tubifex and corresponding changes in the concentration of a metallothionein-like protein. Environmental Toxicology and Chemistry, 2004, 23: 76-85.

[26] Liang LN, He B, Jiang GB et al. Evaluation of mollusks as biomonitors to investigate heavy metal contaminations along the Chinese Bohai Sea. Science of the Total Environment, 2004, 324: 105-113.

[27] Li Lina, Chen Zhenlou, Xu Shiyuan et al. The environment of $\mathrm{Cu}, \mathrm{Zn}, \mathrm{Pb}, \mathrm{Cr}$ and Ni mollusk in the Tidal flat of Yangtze Estuary. Journal of East China Normal University (Natural Science), 2005, (3) : 65-70( in Chinese with English abstract). [ 李丽娜, 陈振楼, 许世远等. 铜锌铅铬镍重金属在长江口滨岸带软体动物体内的富集. 华东师范大学学 报：自然科学版, 2005,(3): 65-70.] 